\title{
$B$ Meson Tagging and Identification with the HERA-B RICH
}

\author{
T. Živko \\ J. Stefan Institute, Jamova 39, 1000 Ljubljana, Slovenia \\ E-mail: T'omi.Zivko@ijs.si!
}

ABSTRACT: The RICH counter for the HERA-B experiment is in operation. Its measured performance is in accord with expectations. The identification and tracking capabilities of the counter are studied using Monte Carlo (MC) simulated events.

The HERA-B experiment (figure $\left.\underline{1}_{1}^{-1}\right)$ is designed primarily to search for $C P$ violation in decays of $B$ mesons that are produced in interactions of $920 \mathrm{GeV}$ protons with an internal wire target [i1].1. Identification of charged particles over a wide range of momenta is one of the basic requirements for the experiment.

The RICH detector has been designed to separate kaons from pions and protons over most of their momentum spectrum $(3-80 \mathrm{GeV} / \mathrm{c})$. The high center-of-mass energy of events results in a large number of particles that radiate Cerenkov photons in the RICH counter. This number becomes even larger due to multiple events (typically 4) on target wires in every bunch crossing.

The optical system of the RICH ensures that hits due to photons radiated by each particle form rings on the photon detector. The ring radius is related to the Cerenkov angle, which in turn depends on the velocity of the particle. The mass of the particles can then be deduced using measurements of momentum from the tracking system and velocity from the RICH. In this paper, we shall first describe the RICH counter and then discuss its expected performance as a particle identification and tracking device.

\section{The RICH Counter}

The RICH counter (figure ator vessel, mirror system and photon detectors. The radiator vessel is filled with $108 \mathrm{~m}^{3}$ of $C_{4} F_{10}$.
The radiator gas combines relatively high refractive index $\left(n-1=1.35 \times 10^{-3}\right)$ and low dispersion $\left(\Delta n / \Delta E(3.3 \mathrm{eV})=3 \times 10^{-5}(\mathrm{eV})^{-1}\right)$. Its average thickness is $2.7 \mathrm{~m}$. For this choice of the radiator, the Cerenkov angle for ultrarelativistic particles is $51.5 \mathrm{mrad}$. A system of spherical and planar mirrors reflect Čerenkov photons to the photon detectors which are situated outside of the particle flux. Regions of higher hit occupancy on the photon detectors are covered with $9 \times 9 \mathrm{~mm}^{2}$ pads, while in the regions of the lower occupancy, $18 \times 18 \mathrm{~mm}^{2}$ pads are used. The corresponding errors on measurements of the Čerenkov angle for single photons are $\sigma_{\theta}(9 \times 9)=$ $0.68 \mathrm{mrad}$ and $\sigma_{\theta}(18 \times 18)=1.03 \mathrm{mrad}$, respectively. A typical event picture of the photon detector is shown in figure $\overline{\underline{3}} \mathbf{r}$

From independent measurements of the efficiency and transmission of components of the counter, we expect an average of 31 detected photons for ultrarelativistic particles [2]. This number is confirmed in measurements of Cerenkov rings $[\overline{3}, \overline{3}, \overline{2}]$. This suggests that our understanding of the counter is satisfactory. As a consequence, there is a firm basis for the MC predictions of counter performance as an identification and tracking device, discussed in what follows.

\section{2. $B$ Meson Tagging with the RICH}

A measurement of $C P$ violation is most promising in the decay chain $B^{0} \rightarrow J / \psi K_{S}^{0}$. The flavour 

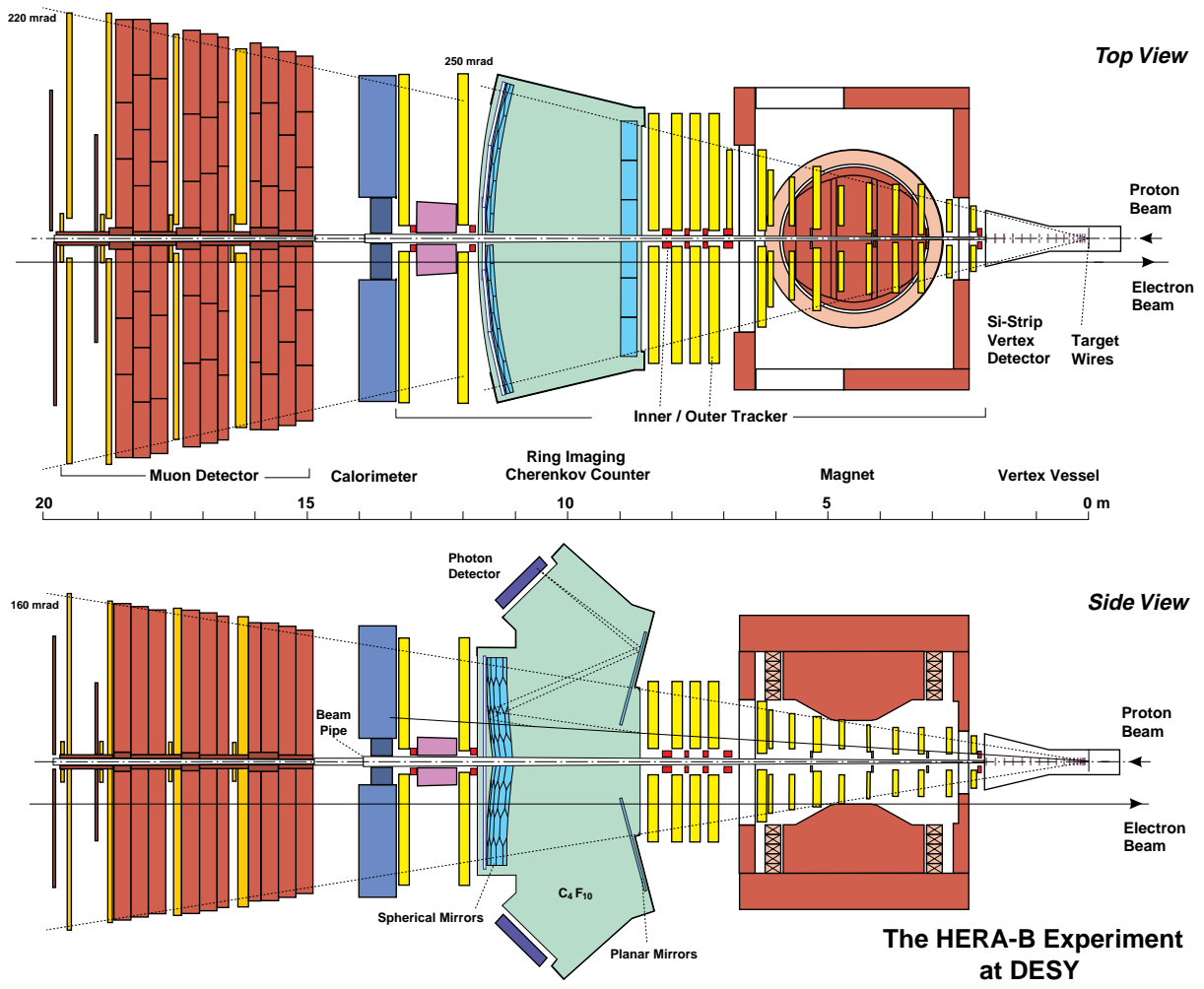

Figure 1: Two views of the HERA-B spectrometer.



Figure 2: The RICH Counter.

of the decayed B meson is deduced from the flavour of the associated produced $B$. Flavour tagging uses the fact that it is more probable for a $B^{0}$ meson to have a positive than negative charged kaon among its decay products. Most of the particles produced at the interaction zone are pions, so it is of crucial importance to separate kaons from pions.

The momentum distribution of tagging kaons as obtained using the MC simulation, is shown in figure $\overline{4}, 4$, while the expected kaon identification efficiency is presented in figure $\overline{1} .1 .50$ can conclude that identification is particularly successful in the momentum region covered by the tagging kaons.

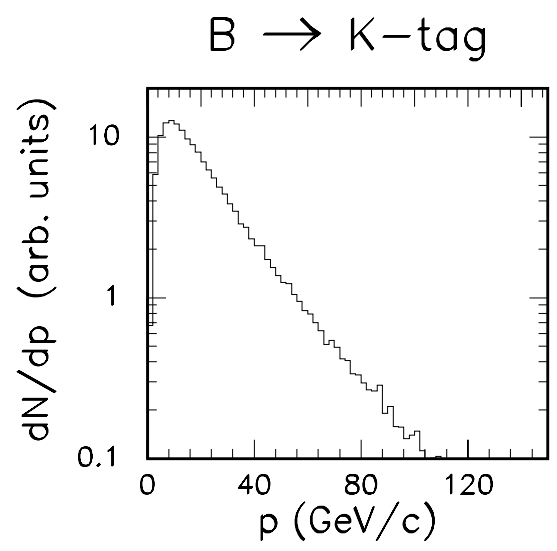

Figure 4: Momentum spectrum of tagging kaons as obtained from MC events. 

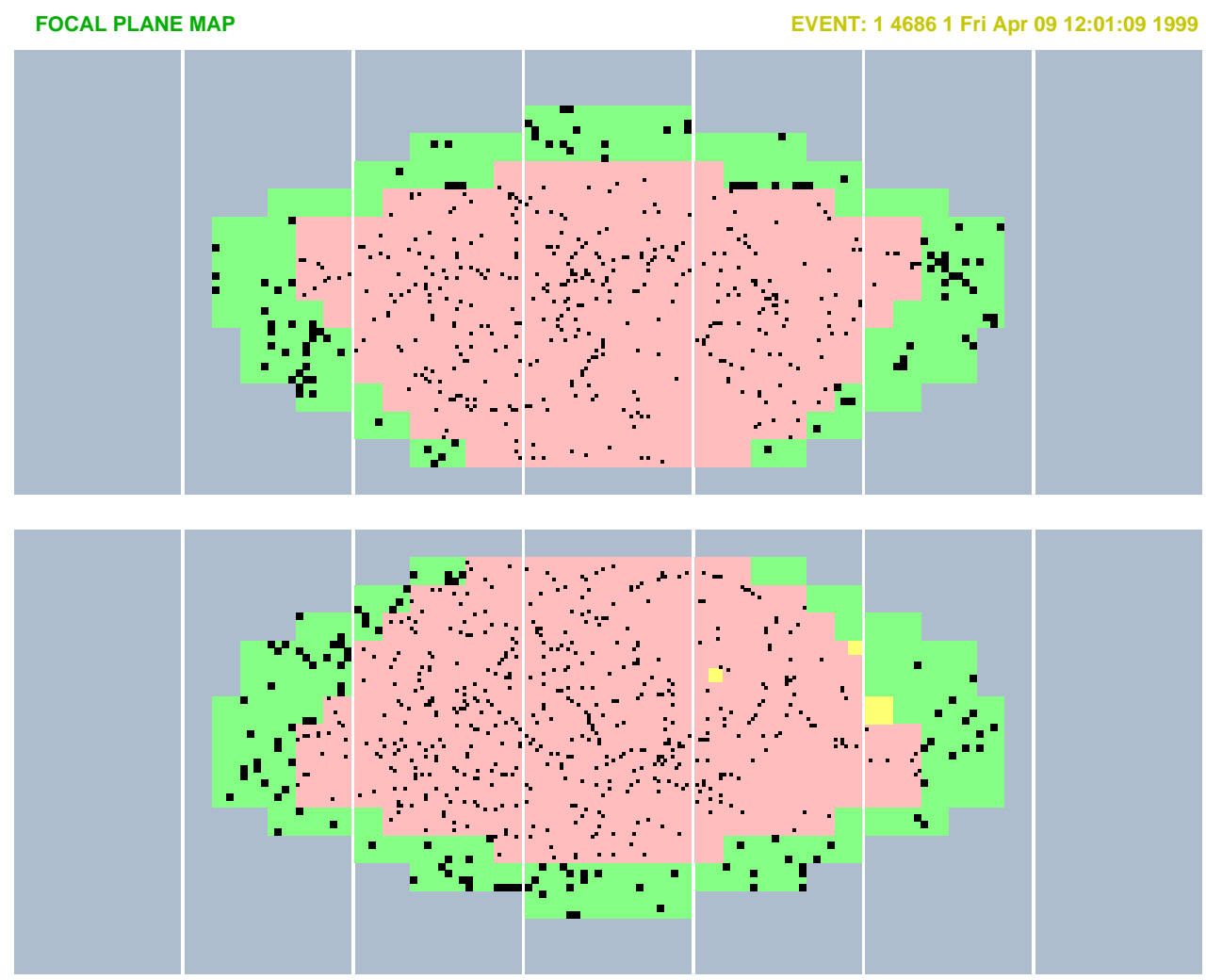

run04686_file001.daq

Figure 3: A measured event with 1117 hits. The figure shows pattern recorded by the the upper and the lower photon detector. Both detectors consist of 7 supermodules covered with $9 \times 9$ (green colour) and $18 \times 18$ $\mathrm{mm}^{2}$ pads (red colour). Yellow colour denotes pads that are not installed. The size of the hit square indicates the size of the pad. Note that supermodules at the edge of each detector are not covered with pads.

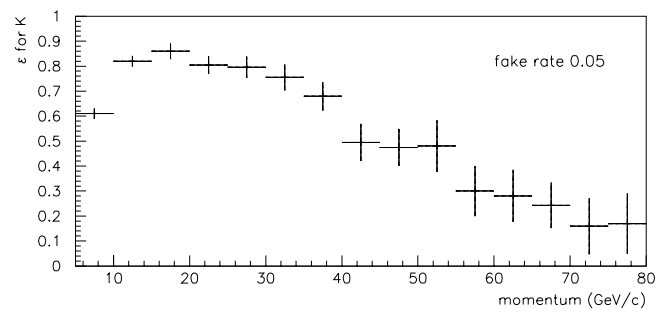

Figure 5: Kaon identification: expected efficiency at $5 \%$ pion false identification probability, obtained from MC events.

\section{Separation between electrons and photons}

The goal of the 1999 HERA-B run is to measure the beauty production cross section via ob- servation of secondary vertices of $J / \psi$ from $b \rightarrow$ $J / \psi X$. In this running period, the RICH counter, calorimeter and vertex detector are ready, while only a small part of the tracking system is installed. Information from the calorimeter is sufficient for the determination of the energy of electrons (and positrons) from $J / \psi$ decays. However, the calorimeter cannot separate photons from electrons and this leads to a non-negligible background underneath the $J / \psi$ peak. In this situation, the RICH can be used for rejection of photons, because only charged particles produce Cerenkov rings. In the case when the HERA$\mathrm{B}$ experiment is operated with the magnetic field switched off, the trajectory of electron candidates is taken to be a straight line connecting the interaction zone with the place of the calorimeter hit. Having the direction of the electron candidate we determine the centre of the assumed 
ring. The quantitative analysis of this picture is performed using a likelihood method. The likelihood of observing the measured hits around the assumed ring centre is calculated for both the electron (Čerenkov ring + random background) and photon (only random background) hypotheses. Here we exploit the fact that electrons from the $J / \psi$ decay are ultrarelativistic and have the same (saturated) Čerenkov angle (51.5 mrad). The procedure was tested on MC events.
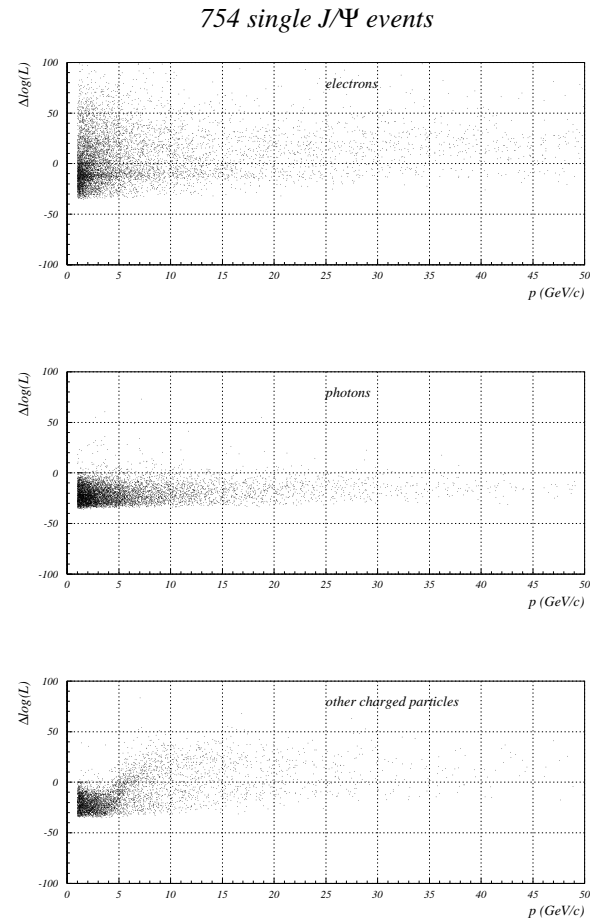

Figure 6: The log-likelihood difference between the electron and photon hypotheses for electrons (top), photons (middle) and all other charged particles (mainly pions, bottom). The distributions are obtained from MC events.

In figure $\overline{6}_{v}^{\prime \prime}$ the difference, $\Delta \log L$, between the likelihoods for the electron and photon hypotheses is plotted for photons, electrons and other charged particles. Electron candidates having $\Delta \log L>0$ were regarded as electrons. It was found that about $80 \%$ of electrons originating from the interaction zone fulfil this cut. The invariant mass distribution of $e^{+} e^{-}$pairs from simulated events containing a $J / \psi$ is shown in figure $\overline{1}$,

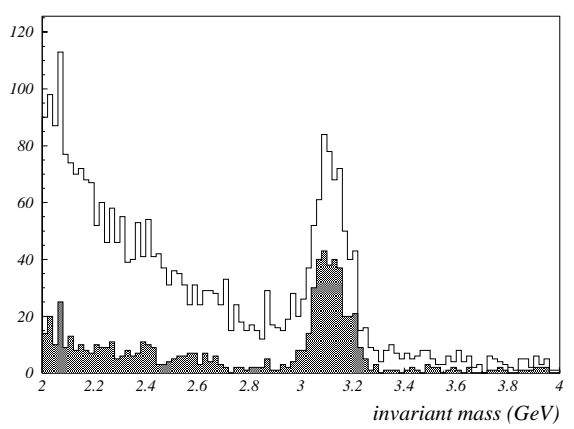

Figure 7: Invariant mass of pairs of MC generated calorimeter clusters for events containing $J / \psi \rightarrow$ $e^{+} e^{-}$decays. Open histogram: no cuts, full histogram: both clusters are identified in the RICH as originating from electrons.

The same idea for separating electrons from photons can be applied in the case of the magnetic field switched on. However, minor complications arise because in this case the magnetic field influences the charged particle trajectory. In a simplified picture (see figure $\overline{8}_{1}^{-1}$ ), the magnetic field bends the trajectory at a single point. The corresponding angles $\phi$ and $\delta$ can be parametrized by $\phi=0.635 / p(p$ in $\mathrm{GeV} / \mathrm{c})$ and $\delta=0.24 / p(p$ in $\mathrm{GeV} / \mathrm{c})$, where $p$ is the momentum determined from the energy deposited in the calorimeter. In this way, two solutions are obtained because we do not know the sign of charge of the particle under consideration. A search is performed for a ring on both the electron as well as on the positron side. This does not cause a problem because the two centres are well separated. This procedure also enables us to reject calorimeter hits produced by charged hadrons, because hadrons leave only a part of their energy in the calorimeter. Correspondingly, we do not reconstruct their momentum correctly, and therefore search for rings centred in the wrong place.

\section{The RICH as a Tracking Device}

The RICH can also operate as a stand alone tracking device. Several ring finding algorithms have been developed to find rings without information on the position of the ring centre. From 


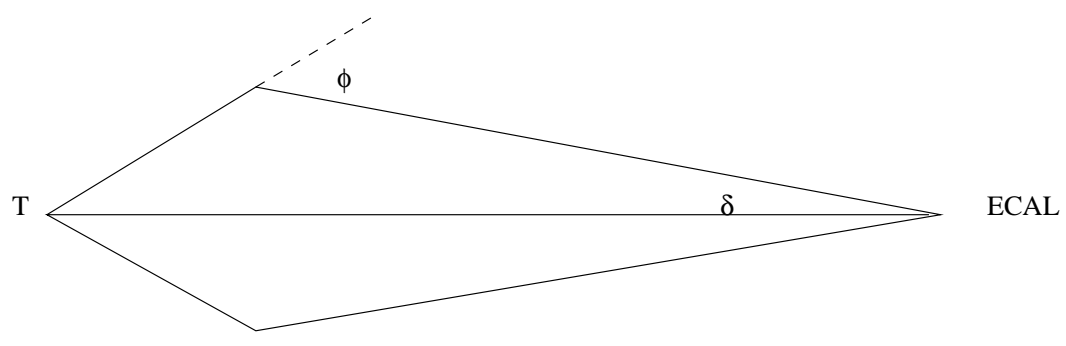

Figure 8: A simplified picture of the influence of the magnetic field on the trajectory of charged particles. By $\mathrm{T}$ we denote the wire target, by $\phi$ the bending angle in the magnet, and $\delta$ the deviation of the track direction from the straight line connecting the calorimeter impact point and the target. The two idealized trajectories correspond to two signs of charge.

the rings found on the photon detector, track directions are deduced and matched in the nonbending plane with the track candidates from the silicon vertex detector. The track momentum can then be calculated from the deflection in the bending plane. The momentum resolution for MC generated tracks is shown in figure $\overline{\underline{9}}_{r}^{1}$. The matching efficiency for low multiplicity events is found to be about $70 \%$.

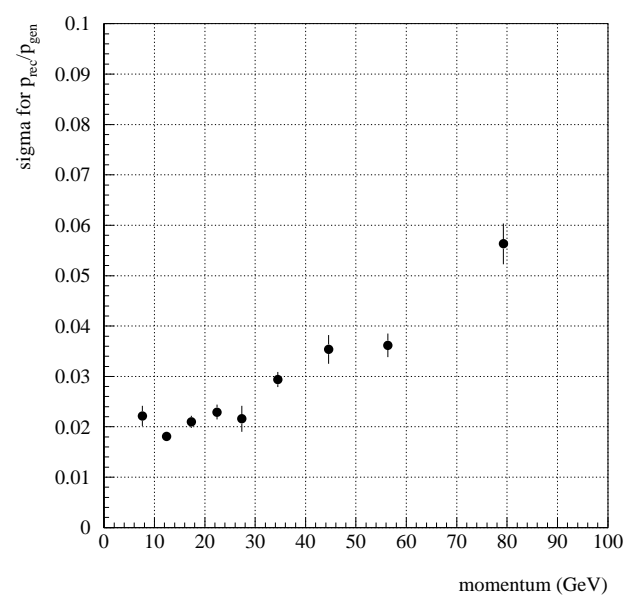

Figure 9: Momentum resolution for MC generated tracks with matched RICH and vertex detector segments.

\section{References}

[1] T. Lohse et al., Proposal for HERA-B, DESY PRC-94/02, May 1994

[2] D. Škrk, Calibration of the Ring Imaging Čerenkov Counter of the HERA-B Spectrometer, Doctoral Thesis, University of Ljubljana, Ljubljana, Slovenia, (1999)
[3] S. Korpar et al., Nucl. Instr. Meth. A 433 (1999) 128 